\title{
Relação entre características empresariais e fatores da transferência de conhecimentos entre projetos de produto
}

\author{
Alejandro Germán Frank ${ }^{\mathrm{a} *}$, José Luis Duarte Ribeiro ${ }^{\mathrm{b}}$, \\ Márcia Elisa Echeveste ${ }^{c}$, Carla Schwengber ten Caten ${ }^{\mathrm{d}}$ \\ a*agerfrank@producao.ufrgs.br, UFRGS, Brasil \\ bribeiro@producao.ufrgs.br, UFRGS, Brasil \\ 'echeveste@producao.ufrgs.br, UFRGS, Brasil \\ dtencaten@producao.ufrgs.br, UFRGS, Brasil
}

\begin{abstract}
Resumo
Este artigo apresenta uma análise das relações existentes entre as características das empresas e a importância que estas atribuem aos fatores que influenciam na transferência de conhecimentos (TC) entre projetos de produtos. A análise é baseada num levantamento (survey) realizado com 58 empresas no Brasil. As caracteristicas estudadas foram: (i) tamanho da empresa; (ii) tamanho da equipe de projetos; (iii) localização das equipes; (iv) número de projetos simultâneos; (v) tempo de duração dos projetos; (vi) grau de inovação; e (vii) complexidade dos projetos. Através de uma análise de aglomerados, foi identificado que as características (ii), (iv) e (vii) são significativas na determinação das importâncias atribuídas aos fatores da TC. Os resultados apontam que as empresas que possuem um perfil de gestão mais complexo enfatizam os fatores de influência pertencentes ao ambiente externo. Já empresas com um perfil de gestão mais simples atribuem maior importância aos fatores internos da empresa.
\end{abstract}

Palavras-chave

Transferência de conhecimentos. Desenvolvimento de produtos. Equipes de projetos. Características empresariais.

\section{Introdução}

As empresas que investem em desenvolvimento de produtos têm um recurso-chave que precisam gerenciar: o conhecimento de suas equipes. lsso se deve a que cada projeto do processo de desenvolvimento de produtos é intensivo na criação de novos conhecimentos, os quais podem ser reutilizados em projetos subsequentes (ZHENGFENG; JINFU; YAN, 2007; RAMESH; TIWANA, 1999). Esse processo de reutilização do conhecimento em outros projetos é denominado transferência de conhecimentos (TC) entre projetos de produto. A TC tem como finalidade melhorar a capacidade da organização de executar suas atividades e capitalizar as experiências passadas das equipes (CUMMINGS; TENG, 2003; DAVENPORT; PRUSAK, 1998; SZULANSKI, 2000; SÖDERQUIST, 2006; HSU, 2008). A TC, quando gerenciada adequadamente, contribui para melhorar o desempenho global do processo de desenvolvimento de produtos (PDP), visto que cada novo projeto pode aproveitar as experiências que as equipes adquiriram em projetos anteriores (MARSH; STOCK, 2006).

No PDP existem diferentes fatores que influenciam a capacidade de TC entre diferentes equipes de projetos (WATSON; HEWETT, 2006; CUMMINGS; TENG, 2003). Para gerenciar a TC entre as equipes, é importante entender quais são esses fatores e como os mesmos atuam em cada empresa em particular. Nesse sentido, um estudo realizado por Frank, Ribeiro e Echeveste (2011) apresenta uma taxonomia dos fatores de influência na TC, baseada numa abordagem sociotécnica. Essa taxonomia classifica os fatores em quatro subsistemas: pessoas, tecnologias, organização do trabalho e ambiente externo.

Mesmo conhecendo quais os fatores que podem influenciar na TC entre os projetos de produto, deve-se considerar que cada empresa possui uma realidade específica, na qual esses fatores podem ter diferentes importâncias no desenvolvimento de produtos. 
As características de cada contexto empresarial podem determinar quais fatores são decisivos para a capacidade de TC (DU; Al; REN, 2007). Associar as características das empresas com um determinado grupo de fatores de influência na TC é um estudo relevante, pois contribui para entender e aprimorar os aspectos relativos à integração de conhecimentos entre as equipes de projetos de produtos.

Em razão disso, o objetivo do presente trabalho é explorar as relações existentes entre as características das empresas e a importância que estas atribuem aos diferentes fatores de influência na TC entre projetos de produtos. Com isso, busca-se identificar se existem características no perfil das empresas que levam a priorizar alguns fatores específicos da TC, permitindo estabelecer quais fatores são determinantes para 0 sucesso da TC em cada contexto empresarial específico.

0 trabalho apresenta um estudo de levantamento (survey) conduzido junto a empresas nacionais e multinacionais que operam no Brasil. Os resultados foram tratados por meio de uma análise de aglomerados, que permite estudar as relações entre o perfil dos respondentes e a importância atribuída aos diferentes fatores da TC. Os resultados obtidos trazem contribuições tanto para o meio acadêmico como para o meio empresarial. Do ponto de vista acadêmico, o trabalho permite ampliar o entendimento da maneira como operam os fatores de influência na TC entre projetos de produto em diferentes contextos empresariais e quais são as dificuldades e limitações para o sucesso da TC nesses contextos. Por outro lado, do ponto de vista empresarial, os resultados permitem direcionar ações de melhoria da TC entre projetos, focalizando especificamente nas necessidades particulares de cada empresa.
Este trabalho está organizado em seis seções. Após esta introdução, a segunda seção apresenta uma revisão da literatura referente aos fatores de influência na TC entre projetos de produto, enquanto a terceira seção discute tipologias empresariais e seu relacionamento com a TC. A quarta seção mostra o método de levantamento dos dados. Na quinta seção, descreve-se a análise dos dados. Por fim, a sexta seção discute os resultados e apresenta as conclusões do trabalho.

\section{Fatores de influência na TC entre os projetos de produto}

Diversas características do ambiente do desenvolvimento de produtos podem influenciar a capacidade de transferir conhecimentos entre as equipes envolvidas em diferentes projetos de produto. Frank, Ribeiro e Echeveste (2011) realizaram uma análise taxonômica que classifica os fatores de influência na TC em características comuns, dentro de uma estrutura de quatro subsistemas do desenvolvimento de produtos. Esses subsistemas estão baseados nos estudos de ambientes sociotécnicos realizados por Hendrick e Kleiner (2001) e ampliados posteriormente por Guimarães (2009). A análise taxonômica de Frank, Ribeiro e Echeveste (2011) foi realizada por meio de uma pesquisa qualitativa, utilizando a técnica de grupos focados com especialistas acadêmicos e profissionais da área de desenvolvimento de produtos.

Conforme é apresentado no Quadro 1, a taxonomia proposta por Frank, Ribeiro e Echeveste (2011) é organizada nos seguintes subsistemas: (i) subsistema pessoal, que considera características das equipes, como profissionalismo, características demográficas e aspectos

Quadro 1. Taxonomia dos fatores da TC.

\begin{tabular}{|c|c|c|}
\hline $\begin{array}{l}\text { Subsistemas } \\
\text { sociotécnicos }\end{array}$ & Caracteristica dos fatores & Fatores \\
\hline \multirow{5}{*}{ Pessoas } & \multirow{2}{*}{$\begin{array}{l}\text { Fatores do } \\
\text { indivíduo }\end{array}$} & Competências técnicas e humanas \\
\hline & & Motivação e interesses individuais \\
\hline & \multirow{3}{*}{$\begin{array}{l}\text { Fatores do } \\
\text { grupo }\end{array}$} & Cultura e clima organizacional \\
\hline & & Estratégias de recursos humanos \\
\hline & & Liderança e estratégias organizacionais (foco nas pessoas) \\
\hline \multirow{4}{*}{ Tecnologias } & \multirow{3}{*}{$\begin{array}{l}\text { Infraestrutura } \\
\text { tecnológica }\end{array}$} & $\mathrm{Tl}$, comunicação e integração de bases de dados \\
\hline & & Acessibilidade dos usuários às tecnologias e bases de dados \\
\hline & & Equipamentos para o desenvolvimento \\
\hline & $\begin{array}{l}\text { Infraestrutura } \\
\text { física }\end{array}$ & Disposição do ambiente físico e adequação da infraestrutura \\
\hline \multirow{4}{*}{$\begin{array}{l}\text { Organização } \\
\text { do trabalho }\end{array}$} & \multirow{2}{*}{$\begin{array}{l}\text { Estratégias do desenvolvimento } \\
\text { de produtos }\end{array}$} & Liderança e estratégias organizacionais (foco na gestão dos projetos) \\
\hline & & Estratégias dos projetos \\
\hline & \multirow{2}{*}{$\begin{array}{l}\text { Práticas do desenvolvimento de } \\
\text { produtos }\end{array}$} & Organização da estrutura e atividades dos projetos \\
\hline & & Utilização de métodos e ferramentas de desenvolvimento de produtos \\
\hline \multirow{4}{*}{ Ambiente externo } & \multirow{2}{*}{ Relacionamento com o meio } & Relacionamento com fornecedores e outras empresas \\
\hline & & Relacionamento com centros de pesquisa \\
\hline & \multirow{2}{*}{ Influência do contexto } & Politicas governamentais \\
\hline & & Formação das pessoas e cultura da região \\
\hline
\end{tabular}

Fonte: adaptado de Frank, Ribeiro e Echeveste (2011). 
psicossociais. Nesse subsistema observam-se fatores relacionados aos indivíduos e ao grupo de trabalho; (ii) subsistema tecnológico, que considera elementos do ambiente físico e das características do trabalho, como equipamentos, ferramentas, automatização, sistemas de informação e infraestrutura; (iii) subsistema organização do trabalho, que envolve a forma em que foi projetado o trabalho, grau de centralização e formalização, estratégias e as práticas utilizadas nas atividades dos projetos; e (iv) subsistema ambiente externo, no qual se consideram aspectos externos como o ambiente socioeconômico, a educação, a política, características culturais e legais.

A premissa que norteia o desenvolvimento deste trabalho é que os fatores descritos anteriormente podem apresentar diferentes prioridades em função de distintas características empresariais. Assim sendo, a seguir apresentam-se as hipóteses do trabalho sobre as possíveis características que poderiam determinar a importância atribuída aos fatores acima descritos.

\section{Tipologia das empresas e seu relacionamento com a TC}

Existem características do perfil de uma empresa que podem estar associadas à importância atribuída aos fatores da TC. Uma dessas características é o tamanho da empresa, que é uma variável que afeta o desempenho do desenvolvimento de produtos, uma vez que a realidade entre empresas pequenas e grandes é diferente em termos de investimentos em inovação e desenvolvimento de novos produtos (COHEN; KLEPPER, 1996). Isso pode acarretar também em diferentes prioridades para o aprendizado e compartilhamento de conhecimento. Por esse motivo, trabalhos como os de Du, Ai e Ren (2007) e Marsh e Stock (2006) consideram o tamanho das empresas como um fator no estudo da TC. No caso de Du, Ai e Ren. (2007), esses autores consideram o tamanho da empresa como uma variável moderadora na modelagem do impacto da TC no desempenho da empresa. Por outro lado, Marsh e Stock (2006) analisaram a utilização de conhecimentos passados em empresas pequenas e grandes. Os resultados desses autores revelaram que as empresas pequenas priorizaram mais do que as grandes a utilização de conhecimentos passados nos novos projetos. Além disso, existem outras pesquisas (por ex.: KAMINSKI; OLIVEIRA; LOPES, 2008; HUANG; CHANG; HENDERSON, 2008; ADENFELT; LAGESTRÖM, 2006; GUPTA; GOVINDARAJAN, 2000; MINBAEVA et al., 2003) que estudaram a TC em contextos específicos para um determinado tamanho de empresas. Em todas essas pesquisas se reconhece que o tamanho da empresa pode levar a diferentes abordagens da TC. Dessa maneira, considera-se a seguinte hipótese:

- H1: 0 tamanho das empresas é uma característica associada à importância atribuída aos diferentes fatores de influência na TC.

0 tamanho das empresas está relacionado com o tamanho das equipes de projetos. Esse é outro aspecto que precisa ser considerado. Trabalhos como os de Akgün, Dayan e Benedetto (2008), Sarin e McDermott (2003) e Zellmer-Brun e Gibson (2006) consideraram a influência do tamanho da equipe na integração e TC entre os participantes dos projetos. Segundo Zellmer-Brun e Gibson (2006), equipes maiores têm disponíveis maiores fontes de conhecimento do que equipes menores, mas também apresentam maiores desafios para lidar com a TC. $\grave{A}$ medida que aumenta o tamanho das equipes, a complexidade dos projetos também cresce, o que conduz a maior dificuldade para lidar com a TC entre as equipes (EDMONDSON; NEMBHARD, 2009). 0 estudo de Sarin e McDermott (2003) registrou uma relação negativa entre o tamanho das equipes e o aprendizado no desenvolvimento de produtos. De acordo com esses autores, isso pode ser devido ao fato que, em equipes maiores, é necessário investir maior esforço nas atividades de coordenação, o que pode levar a menor prioridade das atividades que ajudam ao aprendizado. Portanto, considera-se que:

- H2: 0 tamanho das equipes de projetos é uma característica associada à importância atribuída aos diferentes fatores de influência na TC.

0 compartilhamento do ambiente físico entre equipes de desenvolvimento de produtos foi estudado por vários autores (por ex.: NONAKA, 1994; DAVENPORT; PRUSAK, 1998; NONAKA; TOYAMA; KONNO, 2000; PRENCIPE; TELL, 2001; LAKEMOND; BERGGREN, 2006). Esses autores ressaltam o ambiente físico como um dos principais veículos para que o conhecimento tácito seja compartilhado entre as pessoas de diferentes equipes. Porém, devido à crescente internacionalização das atividades de desenvolvimento de produtos, em muitas empresas as equipes deixaram de ser organizadas da maneira tradicional (em equipes co-localizadas), tornando-se frequente o trabalho através de equipes geograficamente distanciadas, que interagem de maneira virtual (SONG et al., 2007; MONTOYA et al., 2009). Nesse caso, o compartilhamento de experiências é dificultado pela falta de relacionamento face a face entre as pessoas, tornando a comunicação menos fluida e focalizada apenas na solução dos problemas específicos (EDMONDSON; NEMBHARD, 2009; CORSO; PAVESI, 2000; SARKER et al., 2005). Por essa razão, dependendo da forma de organização do trabalho das equipes, diferentes fatores podem ser preponderantes. 
No caso de equipes virtuais, a $\mathrm{Tl}$ tem papel-chave na comunicação entre integrantes das equipes (MONTOYA et al., 2009; EDMONDSON; NEMBHARD, 2009), enquanto em equipes co-localizadas aspectos humanos podem ser prioritários (NONAKA, 1994). Assim sendo, estabelece-se que:

- H3: A proximidade geográfica das equipes de projetos é uma característica associada à importância atribuída aos diferentes fatores de influência na TC.

Autores como Corso, Muffatto e Verganti (1999) e Nobeoka e Cusumano (1997) destacam que o tipo de estratégia adotada em relação à quantidade de produtos a serem desenvolvidos e a possibilidade de utilização de plataformas de produtos influenciam a capacidade e a forma de TC entre equipes. Quando a quantidade de projetos concorrentes é elevada é necessário aproveitar ao máximo componentes de outros projetos para reduzir os tempos de desenvolvimento. Nesse contexto, a TC assume maior relevância. Dessa maneira, podem ser criadas plataformas de produtos que integram elementos e tecnologias de diferentes projetos, sendo para isso importantes os aspectos organizacionais que estruturam a TC (AOSHIMA, 2002; NOBEOKA, 1995; NOBEOKA; CUSUMANO, 1995,1997; CORSO; MUFFATTO; VERGANTI, 1999). Considerando o observado, pode-se estabelecer que:

- H4: A quantidade de projetos concorrentes é uma característica associada à importância atribuída aos diferentes fatores de influência na TC.

O tempo de duração dos projetos é outra característica que pode diferenciar a importância atribuída aos fatores da TC. Projetos mais longos precisam se preocupar mais com a TC, pois as experiências vivenciadas no início podem ser mais facilmente esquecidas (ANTONI; NILSSON-WITELL; DAHLGAARD, 2005; BARTEZZAGHI; CORSO; VERGANI, 1997). Em projetos de longa duração podem ser prioritários alguns fatores da TC, como os sistemas de informações que ajudam a guardar conhecimentos explícitos em um sistema repositório. $\mathrm{Na}$ ausência desses sistemas, pode haver dificuldade para recuperar lições aprendidas nas fases iniciais dos projetos, tornando as equipes vulneráveis à perda da memória dos projetos (ALAVI; LEIDNER, 2001; ANTONI; NILSSON-WITELL; DAHLGAARD, 2005; BOURGEON, 2007). Desse modo, quanto maior for a duração dos projetos, geralmente maior é a necessidade de alguns fatores tecnológicos que facilitem o registro e a recuperação dos conhecimentos explícitos. Portanto, pode-se estabelecer que:

- H5: 0 tempo de duração dos projetos é uma característica associada à importância atribuída aos diferentes fatores de influência na TC.
Por fim, a capacidade e forma de TC também dependem do nível de inovação e complexidade dos projetos de produtos que a empresa decide desenvolver (CHAPMAN; HYLAND, 2004; EDMONDSON; NEMBHARD, 2009). Essas características dependem em certo grau do setor industrial no qual a empresa opera, existindo setores nos quais há maior propensão a desenvolver produtos inovadores e/ou complexos. Empresas que desenvolvem projetos incrementais têm maior facilidade de aproveitamento de conhecimentos técnicos passados (AOSHIMA, 2002), enquanto projetos inovadores têm maior dificuldade de aproveitar experiências passadas por falta de antecedentes com projetos similares. Porém, as empresas que adotam a estratégia de investimento em projetos inovadores geralmente possuem equipes mais abertas ao aprendizado contínuo e às atividades de aprendizado entre os integrantes (HSU, 2008). Por outro lado, a complexidade dos projetos é definida em função do grau de multiplicidade e relacionamentos entre os elementos e itens que compõem a arquitetura dos produtos (CLOSS et al., 2008). Sobre esta característica, algumas pesquisas destacam que quanto maior a complexidade dos projetos, maior é a necessidade de aproveitar os conhecimentos das equipes, fazendo com que a TC assuma maior relevância (CHAPMAN; HYLAND, 2004; AKGÜN et al., 2005; EDMONDSON; NEMBHARD, 2009; KLEINSMANN et al., 2010). Em razão disso, duas hipóteses podem ser estabelecidas:

- H6: 0 grau de inovação dos projetos é uma característica associada à importância atribuída aos diferentes fatores de influência na TC;

- H7: 0 grau de complexidade dos projetos é uma característica associada à importância atribuída aos diferentes fatores de influência na TC.

\section{Método de pesquisa}

Para testar as hipóteses propostas no trabalho, foi realizada uma pesquisa exploratória com abordagem quantitativa. Para tanto, primeiramente foi realizado um levantamento (survey) com empresas nacionais e multinacionais que possuem equipes de desenvolvimento de produtos. Posteriormente, foi processada a análise de aglomerados dos dados levantados. A seguir, são apresentadas essas etapas.

\subsection{Levantamento de dados}

Para o levantamento dos dados foi enviado um questionário que levanta dois tipos de informações: (i) o grau de importância que as empresas atribuem aos fatores de influência na TC apresentados no referencial teórico (Quadro 1) e (ii) as características do 
perfil da empresa respondente (variáveis explicativas). Uma versão resumida do questionário é apresentada no Apêndice A.

Para avaliar o grau de importância atribuído aos fatores da TC, os fatores liderança e estratégias organizacionais focadas nas pessoas e liderança e estratégias organizacionais focadas na gestão dos projetos foram reduzidos a um único fator genérico: liderança e estratégias organizacionais. Isso se deve a que ambos representam diferentes perspectivas do mesmo fator estudado na taxonomia proposta por Frank, Ribeiro e Echeveste (2011). Na avaliação dos fatores da TC utilizou-se uma escala de quatro pontos com o seguinte grau de importância atribuído aos mesmos: (1) pequena; (2) média; (3) alta e (4) muito alta. Foi utilizada uma escala assimétrica, porque se observou em um pré-teste do questionário que os respondentes tendem a atribuir importância elevada a todos os fatores. Assim, as classes estabelecidas permitem maior discriminação entre a real importância dos fatores avaliados.

As variáveis explicativas utilizadas estão associadas às hipóteses estabelecidas neste trabalho, conforme se apresentou no Quadro 2. As variáveis explicativas que utilizaram dados quantitativos foram categorizadas posteriormente. A categorização se realizou dividindo o conjunto de dados levantados em dois níveis, de acordo com as medianas dos valores observados.

Os questionários foram enviados por e-mail a uma lista de 400 contatos de empresas. A amostra foi selecionada por conveniência, utilizando-se a base de dados de contatos do departamento de engenharia de produção de uma universidade federal do Brasil, pertencente à região Sul. A base de dados utilizada contém uma lista de profissionais que participaram em cursos de pós-graduação ministrados pelo departamento dessa universidade. Os contatos pertencem à área de desenvolvimento de produtos de empresas nacionais e multinacionais que operam no país. A maior parte das empresas participantes atuam principalmente na região Sul do Brasil. Dos e-mails enviados, obteve-se um retorno de 58 questionários úteis, que representa uma taxa de resposta de 14,50\%.
A análise de confiabilidade do questionário foi medida com o estimador alfa de Cronbach, obtendo-se um valor de 0,83. Esse valor é considerado aceitável, de forma que não houve necessidade de exclusão de nenhum item do questionário. Nas Tabelas 1 e 2 apresentam-se a composição da amostra em relação ao setor industrial de atuação e os dados demográficos da mesma respectivamente. Na Tabela 2 observa-se que nenhum respondente considerou os projetos com grau de complexidade baixa e, portanto, esse nível de resposta foi excluído das análises posteriores.

\subsection{Procedimentos para a análise de aglomerados}

Com os dados obtidos se realizou uma análise de aglomerados (também conhecida como análise de clusters ou de agrupamentos), que consiste em um conjunto de técnicas multivariadas para identificar estruturas latentes que permitam classificar os respondentes de acordo com a similaridade das respostas fornecidas (MILLIGAN; COOPER, 1987). Essa técnica agrupa os respondentes em função da proximidade das respostas e para isto utilizam-se medidas de similaridade baseadas em distâncias multivariadas (MILLIGAN; COOPER, 1985,1987). Através da análise de aglomerados, buscou-se identificar se existem grupos de respondentes com similaridades no grau de importância atribuída aos

Tabela 1. Composição da amostra em relação ao setor industrial.

\begin{tabular}{lcc}
\hline \multicolumn{1}{c}{ Setor industrial } & Respondentes (N = 58) (\%) \\
\hline Automotiva e aeronáutica & 13 & 22 \\
Metalmecânica & 11 & 19 \\
Projetos de engenharia & 8 & 14 \\
Química e farmacêutica & 7 & 12 \\
Sistemas de logística e transporte & 4 & 7 \\
Eletrônica & 3 & 5 \\
Têxtil & 3 & 5 \\
Construção & 2 & 3 \\
Telecomunicações & 2 & 3 \\
Energia & 2 & 3 \\
Outros & 3 & 5 \\
\hline
\end{tabular}

Quadro 2. Variáveis explicativas das hipóteses estudadas.

\begin{tabular}{|c|c|c|}
\hline Hipótese & $\begin{array}{l}\text { Variável explicativa } \\
\text { (característica da empresa) }\end{array}$ & Tipo de dados \\
\hline $\mathrm{H} 1$ & Número de funcionários da empresa & Dados quantitativos \\
\hline $\mathrm{H} 2$ & $\begin{array}{l}\text { Número de pessoas com envolvimento direto nos projetos } \\
\text { de produtos }\end{array}$ & Dados quantitativos \\
\hline $\mathrm{H} 3$ & $\begin{array}{l}\text { Localização da maior parte dos participantes do } \\
\text { desenvolvimento de produtos }\end{array}$ & $\begin{array}{l}\text { Dados categóricos: (i) equipes co-localizadas; (ii) equipes } \\
\text { dispersas }\end{array}$ \\
\hline $\mathrm{H} 4$ & Média do número de projetos desenvolvidos em simultâneo & Dados quantitativos \\
\hline H5 & Tempo de duração dos projetos & Dados quantitativos \\
\hline H6 & Tipo predominante de projeto quanto ao grau de inovação & $\begin{array}{l}\text { Dados categóricos: (i) projetos de melhoria incremental; (ii) } \\
\text { inovação radical }\end{array}$ \\
\hline $\mathrm{H7}$ & Grau de complexidade dos projetos & Dados categóricos: (i) baixo; (ii) meio; (iii) alto \\
\hline
\end{tabular}


Tabela 2. Dados demográficos da amostra.

\begin{tabular}{|c|c|c|c|c|c|}
\hline Característica & Nível & $\%$ & Característica & Nivel & $\%$ \\
\hline \multirow{2}{*}{$\begin{array}{l}\text { Número de } \\
\text { funcionários }\end{array}$} & Até 500 & 55 & \multirow{2}{*}{$\begin{array}{l}\text { Duração } \\
\text { do projeto }\end{array}$} & Até 12 meses & 67 \\
\hline & Mais de 500 & 45 & & Mais de 12 meses & 33 \\
\hline \multirow{2}{*}{$\begin{array}{l}\text { Número de pessoas } \\
\text { nos projetos de DP }\end{array}$} & Até 30 & 62 & \multirow{2}{*}{$\begin{array}{l}\text { Grau de } \\
\text { Inovação }\end{array}$} & Incremental & 84 \\
\hline & Mais de 30 & 38 & & Radical & 16 \\
\hline \multirow{2}{*}{$\begin{array}{l}\text { Localização das } \\
\text { equipes }\end{array}$} & Co-localizadas & 43 & \multirow{2}{*}{$\begin{array}{c}\text { Grau de } \\
\text { complexidade }\end{array}$} & Média & 66 \\
\hline & Dispersas & 57 & & Alta & 34 \\
\hline \multirow{2}{*}{$\begin{array}{l}\text { Número de } \\
\text { projetos simultâneos }\end{array}$} & Até 10 projetos & 78 & \multirow{2}{*}{\multicolumn{2}{|c|}{ Número de respondentes: $\mathrm{N}=58$}} & \\
\hline & Mais de 10 projetos & 22 & & & \\
\hline
\end{tabular}

diferentes fatores que influenciam na TC, para depois analisar quais as características das empresas que definem esses grupos.

A análise de aglomerados seguiu o procedimento sugerido por Milligan e Cooper (1985) e Hair Junior et al. (1995). A formação dos grupos foi realizada com base no grau de importância atribuída pelos respondentes aos fatores de influência na TC apresentados no Quadro 1. As notas desses fatores foram padronizadas para eliminar possíveis tendências das percepções individuais dos respondentes. A padronização foi realizada de acordo com a Equação 1:

$$
r_{i j}^{*}=r_{i j}+\left(\overline{\bar{X}}-\overline{X_{i}}\right)
$$

Onde: $r_{i j}$ "é a nota padronizada da importância atribuída pelo respondente $i$ para o fator $j$; $r_{i j}$ é a nota de importância atribuída pelo respondente $i$ para 0 fator $j ; \overline{\bar{X}}$ é a média geral de todas as avaliações realizadas para os $n$ fatores estudados; $\overline{X_{i}}$ é a média das notas atribuídas pelo respondente $i$ para os $n$ fatores estudados.

Para a formação dos grupos foi utilizado um método híbrido. Na primeira etapa aplicou-se o procedimento hierárquico (utilizando-se o método de Ward), com medida de similaridade baseada na distância euclidiana. No processo de agrupamento, foram analisadas soluções para a faixa de dois a seis grupos. Na determinação do número apropriado de grupos foram examinados o dendograma e a composição dos grupos obtidos. A análise realizada sugeriu duas alternativas: uma solução de dois e outra de quatro grupos. Dentre as duas alternativas, optou-se pela primeira, pois esta permite realizar melhor comparação dos respondentes, visto que considera um número maior de elementos em cada grupo. Com este número de grupos selecionados, na segunda etapa foi rodada uma nova análise de aglomerados baseada no algoritmo não hierárquico K-Means. Esse algoritmo permite melhorar a solução final dos agrupamentos, e também verificar se os fatores adotados no procedimento hierárquico contribuem significativamente para a formação dos $K$ agrupamentos obtidos (HAIR JUNIOR et al., 1995). Essa última verificação é elaborada por meio da análise de variância (ANOVA).
Uma vez obtidos os grupos foram usados testes de independência para verificar se existem diferenças significativas entre eles no que concerne às características empresariais estudadas. Para categorias em que as composições dos grupos apresentaram mais de 5 observações por nível foi aplicado o teste qui-quadrado de Pearson. Entretanto, para composições que apresentaram 5 ou menos observações por cada nível foi aplicado o teste exato de Fischer.

\section{Resultados da análise de aglomerados}

Os resultados da análise são mostrados nas Tabelas 3 e 4. Na Tabela 3 apresentam-se as médias do grau de importância atribuído a cada fator pelos dois grupos de respondentes e o teste da análise de variância (ANOVA) das diferenças entre essas médias. Nesta tabela, os fatores que mais contribuem na discriminação dos grupos são aqueles com maior valor da estatística F. Observa-se que o Grupo 1 apresenta diferenças significativas em relação ao Grupo 2 na atribuição de maior importância aos fatores dos subsistemas pessoas $\left(X_{2}\right.$ e $\left.X_{5}\right)$, tecnologias $\left(X_{6}\right.$ e $\left.X_{7}\right)$ e organização do trabalho $\left(X_{10}\right.$ e $\left.X_{12}\right)$. Por outro lado, o Grupo 2 atribuiu uma importância significativamente maior que o Grupo 1 aos fatores do subsistema ambiente externo $\left(X_{14} ; X_{15} ; X_{16}\right)$. Observa-se que nesse último subsistema apresentam-se algumas das diferenças mais significativas entre ambos os grupos de empresas. Assim sendo, baseado na Tabela 3, conclui-se que ambos os grupos consideraram mais importante os aspectos internos para a TC do que os aspectos externos à empresa. No entanto, existe um grupo de empresas (Grupo 1) que se diferencia por atribuir uma importância significativamente maior a estes fatores internos. Por outro lado, existe outro grupo de empresas (Grupo 2) que se diferencia por atribuir importância relativamente maior aos fatores externos que influenciam na TC.

0 passo seguinte contemplou testar as hipóteses enunciadas na seção 3. Para tanto, foram usados testes de independência entre os grupos, que permitem verificar se os grupos 1 e 2 diferem estatisticamente no que concerne às características das empresas 
(número de funcionários, número de pessoas no desenvolvimento de produtos etc.). A Tabela 4 resume os resultados obtidos na análise da composição demográfica dos grupos. Na análise do possível efeito das características das empresas, notam-se diferenças significativas entre os dois grupos para as seguintes variáveis explicativas: (i) número de pessoas com envolvimento direto nos projetos de produtos ( $p<0,10)$; (ii) número de projetos desenvolvidos em simultâneo ( $p<0,05)$; e (iii) grau de complexidade dos projetos $(\mathrm{p}<0,05)$. Dessa maneira, os resultados fornecem suporte para as hipóteses H2, H4 e H7. Por outro lado, os resultados não deram suporte para as hipóteses $\mathrm{H} 1, \mathrm{H} 3$, H5 e H6.
Em relação ao número de pessoas no desenvolvimento de produtos (H2), o Grupo 1 possui uma concentração maior de empresas com equipes com até 30 pessoas. Por outro lado, o Grupo 2 apresenta uma distribuição homogênea entre os dois níveis, mas se destaca do Grupo 1 por possuir uma concentração relativa menor para equipes com menos de 30 pessoas e maior para as equipes com mais de 30 pessoas. Em segundo lugar, considerando o número de projetos em andamento (H4), o Grupo 1 se destaca por possuir forte concentração em empresas que gerenciam menos de 10 projetos simultâneos. Já o Grupo 2 apresenta uma concentração relativa ao Grupo 1 um pouco maior de empresas com mais de 10 projetos. Algo similar ocorre com o grau de

Tabela 3. Resultados da análise de aglomerados.

\begin{tabular}{|c|c|c|c|c|}
\hline \multirow{2}{*}{ Sub-sistemas } & \multirow{2}{*}{$\begin{array}{l}\text { Fatores de influência na TC entre os } \\
\text { projetos de produto }\end{array}$} & \multicolumn{2}{|c|}{ Médias } & \multirow{2}{*}{$\begin{array}{l}\text { ANOVA } \\
\text { F-Valor }\end{array}$} \\
\hline & & Grupo 1 & Grupo 2 & \\
\hline \multirow{5}{*}{ Pessoas } & X1- Competências técnicas e humanas & 3,28 & 3,41 & 0,49 \\
\hline & X2- Motivação e interesses individuais & 3,38 & 2,99 & $8,00^{* * * *}$ \\
\hline & X3- Cultura e clima organizacional & 3,10 & 3,22 & 0,52 \\
\hline & X4- Estratégias de recursos humanos & 2,59 & 2,60 & 0,00 \\
\hline & X5- Liderança e estratégias organizacionais & 3,51 & 2,95 & $11,38^{* * *}$ \\
\hline \multirow{4}{*}{ Tecnologias } & X6- T1, comunicação e integração de bases de dados & 3,38 & 2,98 & $7,78^{* * *}$ \\
\hline & X7- Acessibilidade dos usuários às tecnologias e bases de dados & 3,39 & 3,03 & $4,86^{* *}$ \\
\hline & X8- Equipamentos para o desenvolvimento & 3,01 & 2,95 & 0,14 \\
\hline & X9- Disposição do ambiente físico e adequação da infraestrutura & 2,60 & 2,60 & 0,00 \\
\hline \multirow{3}{*}{$\begin{array}{l}\text { Org. do } \\
\text { trabalho }\end{array}$} & X10- Estratégias dos projetos & 3,26 & 2,76 & $10,40^{* * * *}$ \\
\hline & X11- Organização da estrutura e atividades dos projetos & 3,29 & 3,03 & 3,02 \\
\hline & X12- Utilização de métodos e ferramentas de desenv. de produtos & 3,51 & 2,75 & $22,62^{* * *}$ \\
\hline \multirow{4}{*}{$\begin{array}{l}\text { Ambiente } \\
\text { externo }\end{array}$} & X13- Relacionamento com fornecedores e outras empresas & 2,98 & 3,30 & 2,29 \\
\hline & X14- Relacionamento com centros de pesquisa & 2,47 & 3,11 & $12,05^{* * * *}$ \\
\hline & X15- Políticas governamentais & 2,03 & 2,84 & $15,44^{* * *}$ \\
\hline & X16- Formação das pessoas e cultura da região & 1,75 & 2,99 & $50,30^{* * * *}$ \\
\hline
\end{tabular}

*** $p<0,05 ;{ }^{* * *} p<0,01$

Tabela 4. Composição demográfica dos grupos.

\begin{tabular}{|c|c|c|c|c|c|c|}
\hline \multicolumn{2}{|c|}{ Características das empresas } & \multicolumn{2}{|c|}{ Grupo $1(\%)$} & \multicolumn{2}{|c|}{ Grupo $2(\%)$} & \multirow{3}{*}{$\begin{array}{r}\text { p-valor } \\
0,475\end{array}$} \\
\hline \multirow{2}{*}{ Número de funcionários } & Até 500 & 19 & 59 & 13 & 50 & \\
\hline & Mais de 500 & 13 & 41 & 13 & 50 & \\
\hline \multirow{2}{*}{ Número de pessoas nos projetos de DP } & Até 30 & 23 & 72 & 13 & 50 & \multirow{2}{*}{$0,088^{\circ}$} \\
\hline & Mais de 30 & 9 & 28 & 13 & 50 & \\
\hline \multirow{2}{*}{ Localização das equipes } & Co-localizadas & 14 & 44 & 11 & 42 & \multirow{2}{*}{0,912} \\
\hline & Dispersas & 18 & 56 & 15 & 58 & \\
\hline \multirow{2}{*}{ Número de projetos simultâneos } & Até 10 projetos & 29 & 91 & 16 & 62 & \multirow{2}{*}{$0,012^{*}$} \\
\hline & Mais de 10 projetos & 3 & 9 & 10 & 38 & \\
\hline \multirow{2}{*}{ Duração do projeto } & Até 12 meses & 22 & 69 & 17 & 65 & \multirow{2}{*}{0,786} \\
\hline & Mais de 12 meses & 10 & 31 & 9 & 35 & \\
\hline \multirow{2}{*}{ Grau de inovação } & Incremental & 26 & 81 & 23 & 88 & \multirow{2}{*}{0,495} \\
\hline & Radical & 6 & 19 & 3 & 12 & \\
\hline \multirow{3}{*}{ Grau de complexidade } & Média & 25 & 78 & 13 & 50 & \multirow{2}{*}{$0,025^{\circ *}$} \\
\hline & Alta & 7 & 22 & 13 & 50 & \\
\hline & $\mathrm{N}=$ & 32 & & 26 & & \\
\hline
\end{tabular}


complexidade (H7), pois o Grupo 1 novamente apresenta uma concentração maior de empresas com um nível médio de complexidade, enquanto o Grupo 2 tem uma distribuição homogênea para os dois níveis analisados e relativamente superior na concentração de empresas com complexidade alta.

\section{Discussão dos resultados}

A análise de aglomerados dividiu as empresas em dois grupos. 0 Grupo 1 é composto por empresas que possuem um perfil de gestão do PDP mais simples do que o Grupo 2. Considera-se mais simples por estar composto principalmente por equipes menores, com menor número de projetos e de complexidade menor, se comparados com o Grupo 2. Nos resultados, observa-se que ambos os grupos consideraram relevante a maioria dos fatores. Contudo, o Grupo 1 atribuiu uma importância maior do que o Grupo 2 aos fatores internos do PDP, isto é, fatores que compõem os subsistemas pessoas, tecnologias e organização do trabalho. Uma possível explicação para as prioridades deste grupo é que essas empresas ainda precisam lidar com problemas internos de gestão pela falta de maturidade do PDP. Essa suposição pode ser reforçada pelo fato de essas empresas não atribuírem um alto grau de importância ao subsistema ambiente externo, o que pode ser devido à dificuldade que têm para lidar com os aspectos internos. Outra possível explicação para a pouca importância que esse grupo atribuiu ao ambiente externo é que essas empresas não possuem uma visão sistêmica que lhe ajude a esclareçer como os fatores externos podem afetar à TC entre projetos de produto.

Observa-se que o Grupo 2 é mais homogêneo na distribuição de respondentes entre as diferentes categorias, mas possui um número de empresas relativamente maior do que Grupo 1 com um perfil de gestão do PDP mais complexo. Mesmo que esse grupo também tenha considerado importantes os aspectos dos subsistemas pessoas, tecnologia e organização do trabalho, as empresas que o integram não atribuíram um grau de importância tão elevado quanto o primeiro grupo. Uma provável explicação pode ser que este grupo possui maior maturidade nos processos internos de gestão, o que leva a que os fatores internos não sejam considerados tão importantes. Isso pode ser observado, por exemplo, em fatores tais como liderança e estratégias organizacionais, estratégias de gestão dos projetos e utilização de métodos e ferramentas de desenvolvimento de produtos, nos quais esse grupo atribuiu um peso relativamente baixo comparado ao Grupo 1, provavelmente por serem aspectos já adequadamente resolvidos no âmbito do PDP dessas empresas. Por outro lado, esse grupo se interessou mais do que o Grupo 1 pelos fatores externos, o que reforça a ideia anterior, pois, ao terem maior maturidade nos processos internos e terem projetos mais complexos, a preocupação por fontes externas de conhecimentos tende a ser maior. Dessa maneira, essas empresas preocupam-se na busca de integração com centros de pesquisa, os quais podem ajudar a lidar com a complexidade dos seus projetos (BEKKERS; FREITAS, 2008). Além disso, esse grupo também atribui maior importância ao aproveitamento de incentivos governamentais à inovação e à existência de mão de obra qualificada na região onde opera.

Esses resultados são reforçados por pesquisas anteriores reportadas na literatura. Por exemplo, Santamaría, Nieto e Barge-Gil (2009) destacaram a importância das fontes externas de conhecimento para a inovação nos produtos. Nesse sentido, Cohen e Levintal (1990) ressaltam que as capacidades internas do PDP são as que fornecem as condições para que as empresas possam absorver conhecimentos do ambiente externo. Portanto, naturalmente a preocupação primária de uma empresa deveria ser seus processos internos para, posteriormente, concentrar sua atenção na integração com o ambiente externo. Essa necessidade de integração de conhecimentos com o ambiente externo é reforçada quando a complexidade dos projetos é maior, dando lugar a novos paradigmas como, por exemplo, a inovação aberta, que considera o PDP da empresa como um sistema aberto para receber e compartilhar conhecimentos com fontes externas de conhecimento (CHESBROUGH, 2003; ENKEL; GASSMANN; CHESBROUGH, 2009).

Por outro lado, os resultados obtidos não deram suporte a quatro das sete hipóteses estabelecidas. $\mathrm{O}$ tamanho da empresa ( $\mathrm{H} 1)$, associado com a capacidade de investimento que a mesma possui, não apresentou diferenças significativas entre os grupos. Uma possível razão pode ser que o investimento na TC no PDP não dependa diretamente do tamanho da empresa, mas do tamanho da equipe de projetos, que foi uma característica empresarial que apresentou efeito significativo. Assim sendo, mesmo que o tamanho da empresa e o tamanho da equipe sejam duas características que podem ter uma correlação importante, o tamanho da equipe poderia definir melhor os aspectos associados à TC.

Em relação à proximidade geográfica das equipes (H3), não foi possível discriminar os grupos por meio dessa característica. É notório que, para ambos os grupos, o fator ambiente físico e adequação da infraestrutura, que está relacionado com essa característica, teve uma das menores notas de importância. lsso é, portanto, um aspecto que exige maiores estudos. Uma possível explicação é a prioridade 
maior que os entrevistados podem atribuir às fontes de conhecimento explícito, o que faz com que não seja considerado importante o fato de as pessoas estarem no mesmo ambiente. Outra explicação poderia ser que este é um aspecto já superado pelas empresas, fazendo com que elas não o considerem relevante nas prioridades para a TC.

0 tempo de duração dos projetos (H5) e o grau de inovação dos projetos (H6) foram duas hipóteses que tampouco revelaram efeito significativo enquanto variáveis explicativas da importância atribuída aos fatores de influência na TC. Observa-se que, para ambas as variáveis, houve forte concentração de empresas em um dos níveis analisados, o que pode ter dificultado a discriminação dos respondentes através da análise de aglomerados. Assim sendo, trabalhos futuros deveriam tentar abordar setores empresariais nos quais exista um número maior de empresas para ambos os níveis de estudo dessas variáveis explicativas.

\section{Conclusões}

Este trabalho apresentou um estudo das relações existentes entre as características das empresas e a importância que estas atribuem aos diferentes fatores de influência na TC entre projetos de produtos. Observou-se que há três características que diferenciam a importância atribuída aos fatores de TC: o tamanho da equipe de projeto $(\mathrm{H} 2)$; o número de projetos desenvolvidos simultaneamente $(\mathrm{H} 4)$ e a complexidade dos projetos (H7). Essas características afetam significativamente a importância que as empresas atribuem aos fatores de influência na TC.

Os resultados descritos trazem contribuições tanto para o meio acadêmico como para o ambiente empresarial. Do ponto de vista acadêmico, o trabalho permitiu explorar as características das empresas que determinam as diferentes importâncias atribuídas aos fatores de influência na TC. Isso permite ampliar o entendimento a respeito da TC no PDP. A partir disso, futuras pesquisas poderiam aprofundar a investigação da interação entre os fatores analisados, dentro de um determinado contexto de empresas. Por outro lado, do ponto de vista empresarial, os resultados ajudam a identificar quais características devem ser enfatizadas para cada tipo de empresa, permitindo direcionar possíveis soluções para a TC. Assim sendo, futuras pesquisas poderiam desenvolver métodos para a melhoria da TC, considerando as características estudadas no presente artigo. Além disso, conforme se destacou nas discussões do trabalho, as hipóteses que não foram suportadas através dos resultados também apresentam oportunidades para futuras pesquisas. Futuros trabalhos poderiam se concentrar nessas hipóteses e explorá-las em um tamanho maior de amostra, incluindo também outros setores industriais não considerados, a fim de obter maiores evidências sobre as mesmas.

Finalmente, uma das limitações da pesquisa foi que as empresas têm uma vinculação com uma região específica do Brasil, apesar de muitas empresas pesquisadas possuírem atuação nacional e multinacional. Assim sendo, futuras pesquisas poderiam também ampliar o escopo deste trabalho para determinar se existe alguma diferença quando os respondentes pertencem a outras regiões ou, inclusive, a outros países.

\section{Referências}

ADENFELT, M.; LAGESTRÖM, K. Knowledge development and sharing in multinational corporations: The case of a centre of excellence and a transnational team. International Business Review, v. 15, p. 381-400, 2006. http://dx.doi.org/10.1016/j.ibusrev.2006.05.002

AKGÜN, A. E. et al. Knowledge networks in new product development projects: A transactive memory perspective. Information and Management, v. 42, p. 1105-1120, 2005. http://dx.doi.org/10.1016/j.im.2005.01.001

AKGÜN, A. E.; DAYAN, M.; BENEDETTO, A. New product development team intelligence: Antecedents and consequences. Information \& Management, v. 45, p. 221-226, 2008. http://dx.doi.org/10.1016/j. im.2008.02.004

ALAVI, M.; LEIDNER, D. E. Knowledge Management and Knowledge Management Systems: conceptual foundations and research issues. MIS Quarterly Review, v. 25, n. 1, p. 107-136, 2001. http://dx.doi. org/10.2307/3250961

ANTONI, M.; NILSSON-WITELL, L.; DAHLGAARD, J. J. Inter-project improvement in product development. International Journal of Quality \& Reliability Management, v. 22, n. 9, p. 876-893, 2005. http:// dx.doi.org/10.1108/02656710510625194

AOSHIMA, Y. Transfer of system knowledge across generations in new product development: empirical observation from Japanese automobile development. Industrial Relations, v. 41, n. 4, p. 605-628, 2002. http:// dx.doi.org/10.1111/1468-232X.00266

BARTEZZAGHI, E.; CORSO, M.; VERGANI, R. Continuous improvement and inter-project learning in new product development. International Journal of Technology Management, v. 14, n. 1, p. 116-138, 1997. http:// dx.doi.org/10.1504/1JTM.1997.001704

BEKKERS, R.; FREITAS; I. S. B. Analysing knowledge transfer channels between universities and industry: To what degree do sectors also matter? Research Policy, v. 37, p. 1837-1853, 2008. http://dx.doi.org/10.1016/j. respol.2008.07.007

BOURGEON, L. Staffing approach and conditions for collective learning in project teams: The case of new product development projects. International Journal of Project Management, v. 25, p. 413-422, 2007. http:// dx.doi.org/10.1016/j.jproman.2007.01.014

CHAPMAN, R.; HYLAND, P. Complexity and learning behaviors in product innovation. Technovation, 
v. 24, p. 553-561, 2004. http://dx.doi.org/10.1016/ S0166-4972(02)00121-9

CHESBROUGH, H. W. Open Innovation: The New Imperative for Creating and Profiting from Technology. Cambridge: Harvard Business School Publishing, 2003.

CLOSS, D. J. et al. Toward a theory of competencies for the management of product complexity: Six case studies. Journal of Operations Management, v. 26, p. 590-610, 2008. http://dx.doi.org/10.1016/j. jom.2007.10.003

COHEN, W. M.; LEVINTHAL, D. A. Absorptive capacity: a new perspective on learning and innovation. Administrative Science Quarterly, v. 35, p. 128-152, 1990. http://dx.doi. $\operatorname{org} / 10.2307 / 2393553$

COHEN, W. M.; KLEPPER, S. Firm Size and the Nature of Innovation within Industries: The Case of Process and Product RetD. The Review of Economics and Statistics, v. 78, n. 2, p. 232-243,1996. http://dx.doi. org/10.2307/2109925

CORSO, M.; MUFFATTO, M.; VERGANTI, R. Reusability and multi-product development policies: a comparison of approaches in the automotive, motorcycle and earthmoving machinery industries. Robotics and Computer-Integrated Manufacturing, v. 15, n. 1, p. 155-165, 1999. http://dx.doi.org/10.1016/ S0736-5845(99)00010-1

CORSO, M.; PAVESI, S. How management can foster continuous product innovation. Integrated Manufacturing Systems, v. 11, n. 3, p. 199-211, 2000. http://dx.doi.org/10.1108/09576060010320443

CUMMINGS, J. L.; TENG, B. S. Transferring R\&D knowledge: the key factor affecting knowledge transfer success. Journal of Engineering and Technology Management, v. 20 , n. $1-2$, p. 39-68, 2003. http://dx.doi.org/10.1016/ S0923-4748(03)00004-3

DAVENPORT, T. H.; PRUSAK, L. Working Knowledge. Boston: Harvard Business Scholl Press, 1998.

DU, R.; Al, S.; REN, Y. Relationship between knowledge sharing and performance: a survey in Xi'an, China. Expert Systems with Applications, v. 32, p. 38-46, 2007. http:// dx.doi.org/10.1016/j.eswa.2005.11.001

EDMONDSON, A. C.; NEMBHARD, 1. M. Product Development and Learning in Project Teams: The Challenges Are the Benefits. Journal of Product Innovation Management, v. 26, p. 123-138, 2009. http://dx.doi.org/10.1111/ j.1540-5885.2009.00341.x

ENKEL, E.; GASSMANN, 0.; CHESBROUGH, H. Open R\&D and open innovation: exploring the phenomenon. $R \& D$ Management, v. 39, n. 4, 2009. http://dx.doi. org/10.1111/j.1467-9310.2009.00570.x

FRANK, A. G.; RIBEIRO, J. L. D.; ECHEVESTE, M. E. Uma análise dos fatores de influência na transferência de conhecimentos entre projetos de produto. In: CONGRESSO BRASILEIRO DE GESTÃO DE DESENVOLVIMENTO DE PRODUTOS - CBGDP, 8., 2011, Porto Alegre. Anais... IGDP, 2011.

GUIMARÃES, L. B. M. The practice of Ergonomics in the south of Brazil from a sociotechnical perspective. In: SCOTT, P. A. (Org.). Ergonomics in Developing Countries. London: Taylor and Francis, 2009. v. 1.

GUPTA, A. K.; GOVINDARAJAN, V. Knowledge flows between multinational corporations. Strategic Management Journal, v. 21, n. 4, p. 473-496, 2000. http://dx.doi.
org/10.1002/(SICl) 1097-0266(200004)21:4\%3C473::A ID-SMJ84\%3E3.0.C0;2-1

HAIR JUNIOR, J. F. et al. Multivariate data analysis. 4. ed. Ney York: Prentice-Hall International. (1995).

HENDRICK, H. W.; KLEINER, B. M. Macroergonomics: an introduction to work system design. Santa Monica: Human Factors and Ergonomics Society, 2001.

HSU, l-C. Knowledge sharing practices as a facilitating factors for improving organizational performance through human capital: A preliminary test. Expert Systems with Applications, v. 35, p. 1316-1326, 2008. http://dx.doi. org/10.1016/j.eswa.2007.08.012

HUANG, C-M.; CHANG, H-C.; HENDERSON, S. Knowledge Transfer Barriers Between Research and Development and Marketing Groups Within Taiwanese Small- and Medium-Sized Enterprise High-Technology New Product Development Teams. Human Factors and Ergonomics in Manufacturing, v. 18, n. 6, p. 621-657, 2008. http:// dx.doi.org/10.1002/hfm.20130

KAMINSKI, P. C.; OLIVEIRA, A. C.; LOPES, T. M. Knowledge transfer in product development processes: A case study in small and medium enterprises (SMEs) of the metal-mechanic sector from São Paulo, Brazil. Technovation, v. 28, p. 29-36, 2008. http://dx.doi. org/10.1016/j.technovation.2007.07.001

KLEINSMANN, M.; BUIJS, J.; VALKENBURG, R. Understanding the complexity of knowledge integration in collaborative new product development teams: A case study. Journal of Engineering and Technology Management, v. 27, p. 20-32, 2010. http://dx.doi.org/10.1016/j. jengtecman.2010.03.003

LAKEMOND, N.; BERGGREN, C. Co-locating NPD? The need for combining project focus and organizational integration. Technovation, v. 26, p. 907-819, 2006. http://dx.doi.org/10.1016/j.technovation.2005.04.004

MARSH, S. J.; STOCK, G. N. Creating dynamic capability: the role or intertemporal integration, knowledge retention and interpretation. Journal of Product Innovation Management, v. 23, p. 422-436, 2006. http://dx.doi. $\operatorname{org} / 10.1111 / \mathrm{j} .1540-5885.2006 .00214 . x$

MILLIGAN, G. W.; COOPER, M. C. An examination of procedures for determining the number of clusters in a data set. Psychometrika, v. 50, n. 2, p. 159-179, 1985. http://dx.doi.org/10.1007/BF02294245

MILLIGAN, G. W.; COOPER, M. C. Methodology Review: Clustering Methods. Applied Psychological Measurement, v. 11, p. 329-354, 1987. http://dx.doi. org/10.1177/014662168701100401

MINBAEVA, D. et al. MNC knowledge transfer, subsidiary absorptive capacity, and HRM. Journal of International Business Studies, v. 34, p. 586-599, 2003. http://dx.doi. org/10.1057/palgrave.jibs.8400056

MONTOYA, M. M. et al. Can You Hear Me Now? Communication in Virtual Product Development Teams. Journal of Product Innovation Management, p. 139-155, 2009. http://dx.doi.org/10.1111/j.1540-5885.2009.00342.x

NOBEOKA, K. Inter-project learning in new product development. Academy of Management Journal, p. 432-436, 1995.

NOBEOKA, K.; CUSUMANO, M. A. Multiproject strategy and sales growth: the benefits of rapid design transfer in new product development. Strategic Management Journal, v. 18, n. 3, p. 169-186, 1997. http://dx.doi.org/10.1002/ 
(SICl) 1097-0266(199703)18:3\%3C169::AID-SMJ863\%3 E3.0.CO;2-K

NOBEOKA, K.; CUSUMANO, M. A. Multiproject strategy, design transfer, and project performance: a survey of automobile development projects in the US and Japan. IEEE Interactions on Engineering Management, v. 42, n. 4, 397-409, 1995.

NONAKA, 1. A. dynamic theory of organizational knowledge creation. Organization Science, v. 5, n. 1, p. 14-37, 1994. http://dx.doi.org/10.1287/orsc.5.1.14

NONAKA, I.; TOYAMA, R.; KONNO, N. SECl, Ba and Leadership: a Unified Model of Dynamic Knowledge Creation. Long Range Planning, v. 33, p. 5-34, 2000. http://dx.doi.org/10.1016/S0024-6301(99)00115-6

PRENCIPE, A.; TELL, F. Inter-project learning: processes and outcomes of knowledge codification in project-based firms. Research Policy, v. 30, n. 9, p. 1373-1394, 2001. http://dx.doi.org/10.1016/S0048-7333(01)00157-3

RAMESH, B.; TIWANA, A. Supporting Collaborative Process Knowledge Management in New Product Development Teams. Decision Support Systems, v. 27, p. 213-235, 1999. http://dx.doi.org/10.1016/S0167-9236(99)00045-7

SANTAMARÍA, L.; NIETO, M. J.; BARGE-GIL, A. Beyond formal RetD: Taking advantage of other sources of innovation in low and medium technology industries. Research Policy, v. 38, p. 507-517, 2009. http://dx.doi. org/10.1016/j.respol.2008.10.004

SARIN, S.; McDERMOT, C. M. The Effect of Team Leader Characteristics on Learning, Knowledge Application, and Performance of Cross-Functional New Product Development Teams. Decision Sciences, v. 34, n. 4, 2003. http://dx.doi.org/10.1111/j.1540-5414.2003.02350.x

SARKER, S. et al. Knowledge transfer in virtual system development teams: an exploratory study of four key enablers. IEEE Transactions on Professional Communication, v. 48, n. 2, p. 201-218, 2005. http:// dx.doi.org/10.1109/TPC.2005.849650
SÖDERQUIST, K. E. Organizing knowledge management and dissemination in new product development. Long Range Planning, v. 39, n. 5, p. 497-523, 2006. http://dx.doi. org/10.1016/j.lrp.2005.07.004

SONG, M. et al. The effect of IT and Co-location on knowledge dissemination. Journal of Product Innovation Management, v. 24, p. 52-68, 2007. http://dx.doi. $\operatorname{org} / 10.1111 / j .1540-5885.2006 .00232 . x$

SZULANSK1, G. The Process of Knowledge Transfer: A Diachronic Analysis of Stickiness. Organizational Behavior and Human Decision Processes, v. 82, n. 1, p. 9-27, 2000. http://dx.doi.org/10.1006/obhd.2000.2884

WATSON, S.; HEWETT, K. A Multi-Theoretical Model of Knowledge Transfer in Organizations: Determinants of Knowledge Contribution and Knowledge Reuse. Journal of Management Studies, v. 43, n. 2, p. 141-173, 2006. http://dx.doi.org/10.1111/j.1467-6486.2006.00586.x

ZELLMER-BRUHN, M.; GIBSON, C. Multinational organization context: implications for team learning and performance. Academy of Management Journal, v. 49, n. 3, p. 501-518, 2006. http://dx.doi.org/10.5465/ AMJ.2006.21794668

ZHENGFENG, L.; JINFU, Y.; YAN, Z. An empirical study on the effect mechanisms of knowledge management on new product development in aviation industry. In: INTERNATIONAL CONFERENCE ON WIRELESS COMMUNICATIONS, NETWORKING AND MOBILE COMPUTING - WiCom, 2007, Shangai, China. Proceedings... IEEE, 2007.

\section{Agradecimentos}

Este artigo é resultado do projeto "Programa Pesquisador Gaúcho - FAPERGS PqG” n. ${ }^{\circ} 06 / 2010$, processo 1008515. Os autores também agradecem ao CNPq pelo apoio financeiro recebido através de bolsas de pesquisas.

\section{The relationship between company characteristics and influence factors of the inter-product project knowledge transfer}

\section{Abstract}

This paper presents an analysis of the relationship between company characteristics and the importance that companies attribute to the main factors that have influence on inter-product project knowledge transfer (KT). The analysis was based on a survey carried out on a sample of 58 companies that operate in Brazil. The studied characteristics of the companies were: (i) company size; (ii) project team size; (iii) team localization; (iv) number of concurrent projects; (v) project duration; (vi) project innovation and (vii) project complexity. It was possible to identify, using a cluster analysis, that project team size; number of concurrent projects and project complexity are statistically significant regarding the determination of attributed importance for the KT influence factors. The results revealed that companies that have a more complex management profile are more interested in influence factors of the external environment. On the other hand, companies that have a simpler management profile are more interested in influence factors of the internal environment.

\section{Keywords}

Knowledge transfer. Product development. Project teams. business features. 
Apêndice A. Questionário utilizado para o levantamento dos dados

Prezado Gerente / Engenheiro,

Estamos realizando uma pesquisa referente à transferência de conhecimentos (TC) entre equipes de projetos de engenharia/desenvolvimento de produtos (DP). Gostaríamos que você respondesse às perguntas abaixo. Esclarecemos que as informações serão tratadas estatisticamente (sem nenhuma menção a nomes de empresa ou pessoas) e que aqueles que responderem receberão em primeira mão os resultados da pesquisa, com as conclusões referentes aos principais fatores que auxiliam na transferência do conhecimento. 0 preenchimento do questionário pode ser feito em cerca de 15 minutos. A sua contribuição é fortemente agradecida.

Caracteristicas dos projetos de desenvolvimento de produtos:

Setor industrial de atuação da empresa:

Número de funcionários da empresa:

Número de pessoas com envolvimento direto nos projetos de produtos:

Localização dos participantes das equipes de desenvolvimento de produtos:

( ) Local ( )Nacional ( )Internacional

Tipo predominante de projeto, quanto ao grau de inovação:

( )Inovação radical ( )Projetos de melhoria incremental

Tempo de duração dos projetos (em semanas, meses ou anos):

Número de projetos simultâneos em desenvolvimento (em média):

Grau de complexidade dos projetos:

( )Baixo ( )Médio ( )Alto

Importância dos fatores facilitadores da transferência de conhecimento (TC) entre equipes de desenvolvimento de produtos (DP):

1- Importância das competências técnicas e humanas dos integrantes enquanto facilitador da TC entre equipes de DP:

( )Nenhuma ( )Pequena ( )Média ( )Grande ( )Muito Grande

2- Importância da motivação e interesses individuais dos integrantes enquanto facilitador da TC entre equipes de DP:

( )Nenhuma ( )Pequena ( )Média ( )Grande ( )Muito Grande

3- Importância da cultura e clima organizacional da equipe de projeto enquanto facilitador da TC entre equipes de DP:

( )Nenhuma ( )Pequena ( )Média ( )Grande ( )Muito Grande

$\begin{array}{llllll}. & . & . & . & . & . \\ . & . & . & . & . & .\end{array}$

16- Importância da formação das pessoas e cultura da região enquanto facilitador da TC entre equipes de DP:

( )Nenhuma ( )Pequena ( )Média ( )Grande ( )Muito Grande 